Víctor Manuel GÓmez C.*

\title{
UN EXAMEN DE LOS EXÁMENES DE CALIDAD DE LA EDUCACIÓN SUPERIOR (ECAES)**
}

\begin{abstract}
Resumen
El presente texto es una mirada crítica de algunas dimensiones de orden técnico y contextual que inciden sobre el éxito o fracaso de los Exámenes de Calidad de la Educación Superior que, como manifestación de las políticas educativas oficiales, se han empezado a implementar en nuestro país y que pretenden, a través de la medición y la evaluación, lograr un mayor grado de racionalidad, funcionalidad y congruencia entre medios y fines. De allí que este documento, que busca abrir espacio para la reflexión y el debate, se interrogue por los medios utilizados en procura de los fines propuestos, como también por las consecuencias académicas e institucionales que puedan derivarse de la implementación de estos medios y, finalmente, se pregunte por las alternativas de evaluación que, en este caso, podrían proponerse.
\end{abstract}

Palabras clave. Evaluación, exámenes, calidad, educación superior, medición, fines.

\begin{abstract}
The present text is a critical look of some dimensions of the technical and contextual order that on the success and failure of the Higher Education Quality Examinations that, as official educative political manifestation, it was begin to introduce in our country and pretend, through measuring and examination, to get a better degree of rationality, functionality and congruence between midway and purposes. From there that this document, that pursuit to open the reflection and debate space, question about the used midways to obtain the proposed purposes, as well for the academic and institutional consequences that can to be proceed from the implantation of this midways and, finally, it question about the examination alternatives that, in this case, can to be proposed.
\end{abstract}

Key words: Examination, quality, Higher Education, measuring, purposes.

* Instituto de Investigaciones en Educación, Facultad de Ciencias Humanas, Universidad Nacional de Colombia. E-mail- vmgomez@ unal.edu.co

" Artículo recibido el 16 de septiembre de 2003 y aprobado el 31 de octubre de 2003. 
No es asunto de discusión la evidente necesidad que tienen la sociedad, el sector productivo y el Estado, de medir y evaluar la calidad de la educación superior ofrecida en el país. A esta necesidad responde la política de evaluación de la calidad mediante los Exámenes de Calidad de la Educación Superior (ECAES), Decreto 1716, 2001. En desarrollo de dicha política, en el 2003 se efectuarán los ECAES en Ciencias de la Salud (9 programas), Ingeniería, Arquitectura y afines (15 programas) y Ciencias Sociales y Humanas (2 programas). Para el 2006 se espera haber logrado la universalización de los ECAES a todos los programas de formación de nivel superior en el país.

Dada la importancia de esta política, y de sus posibles consecuencias académicas e institucionales, es entonces necesario iniciar el estudio y análisis de un conjunto de dimensiones, tanto de índole técnica como contextual, que inciden decisivamente sobre el éxito o fracaso de dicha política. El propósito de esta propuesta de Examen de los ECAES es entonces lograr mayor racionalidad, funcionalidad y congruencia entre fines y medios. ¿Son los medios utilizados los más adecuados para el logro de los fines propuestos? ¿Qué efectos o consecuencias indeseables pueden derivarse de los medios o instrumentos utilizados? ¿Qué alternativas de medición/ evaluación podrían proponerse?

Dimensiones de análisis:

a) En primer lugar es necesario el análisis de los propósitos buscados: ¿cómo se define y especifica la 'calidad' de la educación ofrecida, y en cada área de formación?

La importancia de este interrogante se deriva del alto grado de heterogeneidad y divergencias; de índole teórica, metodológica y epistemológica; existente entre las diversas áreas de formación, lo que torna muy compleja la tarea de medición y muy arbitraria y anti-técnica la utilización de una sola metodología de evaluación. Esta alta heterogeneidad existe, además, entre diversas instituciones y programas curriculares en una misma área del conocimiento, en razón de las diversas opciones de formación, que son naturales e intrínsecas a la autonomía académica y que no pueden ser homogeneizadas ni estandarizadas, y que por tanto su 'calidad' no puede ser medida o evaluada con el mismo tipo de instrumentos ${ }^{1}$.

En efecto, en cada una de las diversas áreas de formación hasta ahora sujetas a los ECAES (Derecho, Medicina, Ingeniería Mecánica), y también en las de próxima aplicación, existen diversas opciones -o posicionesrespecto a cómo se define y concreta -curricular y pedagógicamente- la 'calidad' y pertinencia externa de la educación ofrecida. Estas opciones difieren desde aquellas que definen la calidad de la educación como la formación de capacidades o competencias generales creativas y divergentes; expresadas en términos de capacidad de aprendizaje autónomo, de conceptualización, argumentación, solución de problemas, desarrollo de prototipos y aplicaciones, capacidad de investigación creativa...; hasta aquellas que valoran la adquisición -y repetición- de informaciones o conocimientos específicos.

Asumiendo que ambas, $\mathrm{u}$ otras opciones, son igualmente legítimas, en cuanto expresión de la natural autonomía académica, no es posible, por tanto, homo-

\footnotetext{
${ }^{1}$ Estas diferencias son comunes entre instituciones y programas, en función de diversas tradiciones intelectuales e institucionales, liderazgos académicos, identidades, paradigmas dominantes, etc. Estas diferencias constituyen la esencia de la autonomía universitaria.
}

geneizar ni unificar estas diversas opciones en un único criterio, norma o definición de calidad, y mucho menos someterlas a una única forma o instrumento de medición o evaluación, aunque esta opción haya sido legitimada por las asociaciones profesionales 0 académicas (como ASCOFAME, ACOFI, ACIEM, ACFA, entre otros). La validez, confiabilidad, deseabilidad, y otras dimensiones técnicas y contextuales de las pruebas, conforman un asunto complejo y multidimensional (multidisciplinario), el que no puede ser reducido ni a la participación de ese tipo de asociaciones (las que sólo confieren legitimidad mas no validez ni confiabilidad) ni a opciones de técnica psicometría.

A pesar de los argumentos anteriores, los ECAES están basados en la pretensión de medir la calidad a través de un único tipo de instrumento que arroje datos empíricos comparativos entre diversos programas de formación. Esta necesidad de comparar y jerarquizar es central en la actual política de evaluación de la calidad de la educación superior. Los resultados deben ser cuantitativos; deben expresarse en datos, indicadores, estadísticas; que permitan la evaluación comparativa y legitimen la toma de decisiones respecto a asignación de recursos. Aquí radica la enorme importancia, el poder político y simbólico, de este tipo particular de evaluaciones de calidad. Es enorme el peligro de sus usos indebidos, arbitrarios, inequitativos, amparados bajo la supuesta objetividad y tecnicidad de los instrumentos. Estos usos, 'políticamente orientados' de los ECAES, puede ser fuente de importantes conflictos entre el Estado y las instituciones de educación superior.

La utilización de un solo tipo de instrumento de medición en de- 
terminada área de formación, implica privilegiar una opción metodológica y epistemológica sobre otras posibles, lo que constituye una opción arbitraria, pues favorece o privilegia a los programas de formación más afines o congruentes con esa opción, y discrimina negativamente en contra de aquellos programas más diferentes o divergentes respecto a la norma o modelo deseable de formación, la que de facto se establece y formaliza mediante la utilización (e imposición) de una particular opción metodológica de evaluación.

b) También es importante el análisis tanto de los resultados empíricos de dichos ECAES, como de sus significados o interpretaciones, y sus implicaciones de política académica. ¿Qué tipo de resultados generan estas pruebas? ¿Puntajes comparativos entre programas e instituciones? ¿O diagnósticos cualitativos de estos programas? ¿Se supone que los puntajes son indicadores válidos de calidad diferencial de la educación ofrecida? ¿En qué se sustenta ese supuesto? ¿Cómo se interpretan los resultados? ¿Qué sucede en casos de puntajes relativamente altos en programas, objetivamente conocidos como de mala o mediocre calidad? ¿Se revisan y evalúan críticamente las pruebas o se legitiman los resultados?

c) Otra dimensión de análisis se refiere a los efectos o consecuencias -sociales e institucionalesde la aplicación de los ECAES y de divulgación de sus resultados:

Algunos de estos efectos posibles tienen que ver con estrategias de instituciones y programas de preparar o 'entrenar' a sus estudiantes para aumentar sus puntajes en los ECAES (práctica además muy común en colegios de secundaria en el
Examen de Estado) sin que el aumento en los puntajes corresponda con una mejor calidad de la educación recibida sino con la estrategia de aumentar el estatus social de la institución, lo que favorece su ventaja competitiva en el mercado de matriculas. En este caso, el propósito formal de los ECAES no se cumple, aunque los instrumentos se apliquen. Prima entonces el formalismo y el instrumentalismo sobre lo sustantivo.

Otra situación posible es el caso de programas cuya opción intelectual y pedagógica sea muy distinta o divergente de aquella que comporta (implícita o explícitamente) el instrumento de medición, lo que puede conducir a la paradoja de bajos puntajes en programas de alta calidad reconocida, y buenos puntajes en programas de menor calidad relativa, en virtud de las limitaciones, o arbitrariedades, inherentes al instrumento utilizado. El instrumento es incompetente para identificar y valorar las diversas innovaciones curriculares y pedagógicas, distintas a la formación tradicional, y que conforman lo sustantivo de la 'calidad' de la educación. En este caso, es evidente la deslegitimación, no sólo del instrumento en particular sino de la política general de medición de la calidad de la educación superior. La mala calidad, o inadecuación, de los instrumentos actúa en contra de los objetivos deseados. Los medios se oponen a los fines.

Comúnmente, este tipo de efectos o consecuencias no son previsibles ni deseados - por lo cual son llamados efectos 'perversos' 0 indeseables- y pueden ser causa eficiente del fracaso, ineficacia o distorsión de los objetivos de políticas o programas, como los ECAES.

d) Otro tema importante se refiere a si la medición o evaluación de la calidad de la educación superior recibida, puede efectuarse con mayor validez, ya sea durante o al final del proceso de formación o, más bien, en el desempeño laboral.

Es necesario señalar al respecto que las evaluaciones, puntajes o notas, logradas durante el periodo de formación, no determinan ni predicen el futuro desempeño laboral del egresado. Es escasa y débil la predictibilidad de éstos en función de los puntajes de pruebas escolares, por las siguientes razones:

* Artificialidad de tests y pruebas escolares: este tipo de pruebas se efectúan en un contexto escolar muy diferente de la complejidad intelectual y contextual del ejercicio profesional.

* Individualización y aislamiento. Los tests o pruebas escolares comúnmente se centran en la respuesta individual diferencial a determinadas preguntas 0 ítems, lo que además de ser un contexto artificial y de escasa predictibilidad laboral, es claramente ajeno al contexto interactivo, comunicativo, colaborativo, propio del desempeño ocupacional en todas las actividades productivas.

* El desempeño laboral del individuo depende de un numeroso y complejo conjunto de factores (como capital social y cultural, rasgos de personalidad, acceso a oportunidades, capacidades comunicativas e interactivas, etc.), las que evidentemente no pueden ser reducidas a puntajes en pruebas escolares, ya señaladas, además, como artificiales, de escaso valor predictivo, y ajenas al contexto del desempeño profesional. Por lo cual éstas son de poca utilidad y pertinencia para la medición de la 'calidad' de la educación recibida, objetivo éste de los ECAES.

En este documento se han presentando brevemente cuatro temas (o 
dimensiones técnicas y contextuales de los ECAES), que afectan significativamente el éxito o fracaso de la política de medición de la calidad de la educación superior:

1. El primero se refiere a la existencia de diversos criterios y posiciones respecto a la definición de qué se entiende por 'calidad' de la educación ofrecida en determinada área del conocimiento (Medicina, Derecho, Ingeniería, Ciencias Sociales, etc.). Estas diversas opciones de formación se expresan en términos de objetivos y prácticas curriculares y pedagógicas muy diferentes y aun antagónicas entre sí, como resultado natural del ejercicio de la autonomía académica en las universidades. Sin embargo, esta heterogeneidad natural es sometida a una sola metodología de evaluación o medición de la calidad de la educación ofrecida (ECAES). Surgen entonces diversos interrogantes sobre la índole arbitraria, anti-técnica e inequitativa de esta única opción de evaluación. ¿Implica ésta una fuerza homogeneizadora de la natural heterogeneidad en las opciones de formación? ¿Es ésta una tendencia deseable 0 , por el contrario, violatoria de la autonomía universitaria? ¿Cuáles serían las implicaciones negativas sobre la calidad de la educación superior, derivadas de la mayor homogeneidad en las opciones de formación? ¿Implica esto mayor conservadurismo y tradicionalismo? ¿Cómo pueden los actuales instrumentos de Ios ECAES reconocer, ponderar y evaluar diversas innovaciones curriculares y pedagógicas, diferentes o divergentes del modelo de formación institucionalizado en los instrumentos? ¿Qué opciones o modelos de formación, entre varios posibles, son privilegiados por esta opción metodológica? ¿Qué otras opciones o modelos son negativamente tratados?
2. El segundo conjunto de interrogantes ya ha sido formulado en el literal b. Se refiere al tipo de datos, resultados o información, producida por los actuales ECAES, y al tipo de análisis, significados e interpretaciones, que de ellos se puede derivar.

3. Otros interrogantes se refieren a diversos efectos imprevistos o no deseados, usualmente negativos, derivados del contexto social e institucional de aplicación de los ECAES. Algunos de éstos son: la homogeneización de objetivos de aprendizaje y de prácticas curriculares; la invisibilidad de innovaciones curriculares y pedagógicas distintas 0 divergentes de la opción 'oficial'; la competencia interinstitucional por el 'puntaje', independientemente de la calidad; la deslegitimación potencial de los instrumentos y, por tanto, de la política.

4. Finalmente, cuándo y cómo puede medirse la calidad de la educación recibida, dadas las limitaciones y la artificialidad de los instrumentos de medición vis-àvis la complejidad y multidimensionalidad del desempeño profesional de los egresados.

Propuesta:

Es indudable la complejidad y multidimensionalidad de los temas e interrogantes aquí planteados sobre los ECAES y la política de medición de la calidad de la educación superior. Esto exige un contexto y proceso de trabajo interdisciplinario, y una estrecha interacción entre el trabajo académico independiente y funcionarios del Estado (Servicio Nacional de Pruebas). También es esencial la capacidad de construir y conservar una mirada o visión de conjunto sobre la complejidad del tema. Cada uno de los temas e interrogantes aquí planteados podrían convertirse en diversos proyectos de investigación. Sin embargo, se co- rre el riesgo de atomización y descontextualización, y la posterior dificultad para integrar, conceptualizar y lograr visión holística de las diversas dimensiones de un mismo problema.

Por consiguiente, suponiendo el natural interés del Estado (ICFES) en el estudio sistemático de esta problemática, se propone la conformación de un equipo académico interdisciplinario, con participación de funcionarios del Servicio Nacional de Pruebas, y que tendría las siguientes responsabilidades:

a) A corto plazo: la identificación y concreción de un conjunto de estudios o proyectos específicos, orientados a la resolución de los interrogantes aquí planteados y de otros que surjan del grupo de académicos propuesto.

b) A mediano plazo: la realización de los estudios propuestos y la integración conceptual y metodológica de sus resultados en dos productos principales:

* Una mejor y más compleja conceptualización de lo que significa medición o evaluación de la calidad de la educación superior, y

* Diversas opciones metodológicas de medición, que den cuenta tanto de la heterogeneidad natural de opciones de formación como de la complejidad y diversidad de las diversas áreas de formación. 